\title{
High-speed stereoscopy of aurora
}

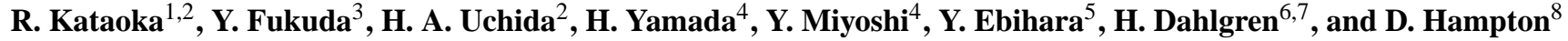 \\ ${ }^{1}$ National Institute of Polar Research, Tokyo, Japan \\ ${ }^{2}$ Department of Polar Science, SOKENDAI, Tachikawa, Japan \\ ${ }^{3}$ Department of Earth and Planetary Science, The University of Tokyo, Hongo, Japan \\ ${ }^{4}$ Institute for Space Earth Environmental Research, Nagoya University, Nagoya, Japan \\ ${ }^{5}$ Research Institute for Sustainable Humanosphere, Kyoto University, Uji, Japan \\ ${ }^{6}$ School of Electrical Engineering, KTH Royal Institute of Technology, Stockholm, Sweden \\ ${ }^{7}$ School of Physics and Astronomy, University of Southampton, Southampton, UK \\ ${ }^{8}$ Geophysical Institute, University of Alaska Fairbanks, Fairbanks, USA \\ Correspondence to: R. Kataoka (kataoka.ryuho@nipr.ac.jp)
}

Received: 29 October 2015 - Revised: 8 December 2015 - Accepted: 21 December 2015 - Published: 18 January 2016

\begin{abstract}
We performed $100 \mathrm{fps}$ stereoscopic imaging of aurora for the first time. Two identical sCMOS cameras equipped with narrow field-of-view lenses $\left(15^{\circ}\right.$ by $\left.15^{\circ}\right)$ were directed at magnetic zenith with the north-south base distance of $8.1 \mathrm{~km}$. Here we show the best example that a rapidly pulsating diffuse patch and a streaming discrete arc were observed at the same time with different parallaxes, and the emission altitudes were estimated as $85-95 \mathrm{~km}$ and $>100 \mathrm{~km}$, respectively. The estimated emission altitudes are consistent with those estimated in previous studies, and it is suggested that high-speed stereoscopy is useful to directly measure the emission altitudes of various types of rapidly varying aurora. It is also found that variation of emission altitude is gradual (e.g., $10 \mathrm{~km}$ increase over $5 \mathrm{~s}$ ) for pulsating patches and is fast (e.g., $10 \mathrm{~km}$ increase within $0.5 \mathrm{~s}$ ) for streaming arcs.
\end{abstract}

Keywords. Ionosphere (auroral ionosphere)

\section{Introduction}

It has been known for a long time that aurora manifests itself in many rapid variations (Stormer, 1955). The rapidly varying aurora has recently been studied as important phenomena to visualize the wave-particle interactions working in the magnetosphere-ionosphere coupled regions (Dahlgren et al., 2008; Kataoka et al., 2011a, b, 2012; Yaegashi et al., 2011; Whiter et al., 2010) and in the magnetosphere (Miyoshi et al.,
2010, 2015a; Nishiyama et al., 2014; Kataoka et al., 2015). The time-varying emission altitude of such various types of rapidly varying aurora contributes especially to understand not only the generation mechanisms but also their possible impact on the atmosphere (Miyoshi et al., 2015b) because the emission altitude contains information of the energy of precipitating electrons and indicates the stopping height of electrons.

Stereoscopy of aurora has also been conducted to determine the emission altitude since the beginning of auroral research (Stormer, 1955), and many sophisticated stereoscopic analyses have been carried out worldwide (e.g., StenbaekNielsen and Hallinan, 1979; Frey et al., 1996; Ivchenko et al., 2005; Whiter et al., 2013). Most recently, Kataoka et al. (2013) performed all-sky stereoscopy of aurora using twin fish-eye DSLR cameras located $8 \mathrm{~km}$ apart, which may give an interesting pathway to open citizen science. The purpose of this paper is to demonstrate another new challenge of "high-speed stereoscopy" of rapidly varying aurora which cannot yet be captured by the daily-use low-cadence DSLR cameras.

\section{Instrumentation}

Two identical sets of Hamamatsu sCMOS cameras (Orca Flash 4.0) were used in this study. Each sCMOS camera was equipped with a NIKKOR $50 \mathrm{~mm}$ F1.2 lens to make the narrow $15.2^{\circ}$ by $15.2^{\circ}$ field of view. SCHOTT RG665 glass filter was used to remove the slow green and red lines. These identical camera systems were installed at Poker Flat Re- 
Poker Flat Camera, lat $=65.12^{\circ} \mathrm{N}$, lon $=-147.47^{\circ} \mathrm{E}$ (red) Aurora Lodge Camera, lat $=65.05^{\circ} \mathrm{N}$, lon $=-147.45^{\circ} \mathrm{E}$ (blue)

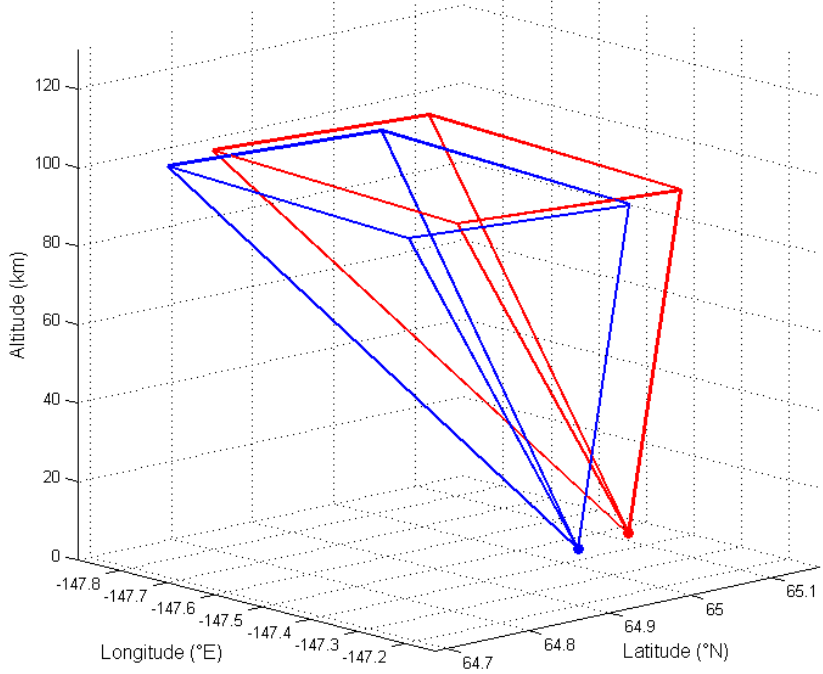

Figure 1. Stereoscopic observation geometry and overlapped fields of view at $100 \mathrm{~km}$ altitude.

search Range (PFRR; 65.1186 N, 147.4328 W; $489 \mathrm{~m}$ ) and at Aurora Borealis Lodge (ABL, 65.0462 N, 147.4512 W, $662 \mathrm{~m}$ ), with the north-south base distance of $8.12 \mathrm{~km}$. Figure 1 shows the observation setting to illustrate the overlapped fields of view from PFRR and from ABL. The sampling rate was $100 \mathrm{fps}$, and 4 by 4 binning was applied to obtain the final images of 512 by 512 pixels. Both cameras are approximately directed toward the magnetic zenith $\left(19^{\circ}\right.$ declination angle, and $77.5^{\circ}$ elevation angle).

\section{Method of analysis}

In order to estimate the emission altitude, we apply essentially the same "optical depth" analysis as documented in detail by Kataoka et al. (2013). Bright stars are used to set the stereoscopic geometry, and then the stars are removed by applying 10 by 10 pixel median filter before the optical depth analysis. The modification parts from Kataoka et al. (2013) are described in this paper. Firstly, we assume a different projection model between two images: simplest horizontal shift is applied because of the standard narrow field of view. Secondly, as shown in Fig. 2, we use only two large template windows of 384 by 160 pixels $\left(10^{\circ}\right.$ by $\left.4.5^{\circ}\right)$, i.e., region of interest (ROI)-1 and ROI-2 for low-latitude part and highlatitude part, respectively, because we are interested more in the time variation of the emission altitude than the spatial resolution in this study. ROI-1 mostly covers the pulsating patches, and ROI- 2 covers the streaming arcs. Finally, we set the altitude resolutions of $2.5,5.0$, and $10.0 \mathrm{~km}$ for the altitude ranges of 70-100, 100-150, and 150-200 km, respectively. The main requirement to apply the analysis is the existence of inhomogeneity of auroral intensity within the fields
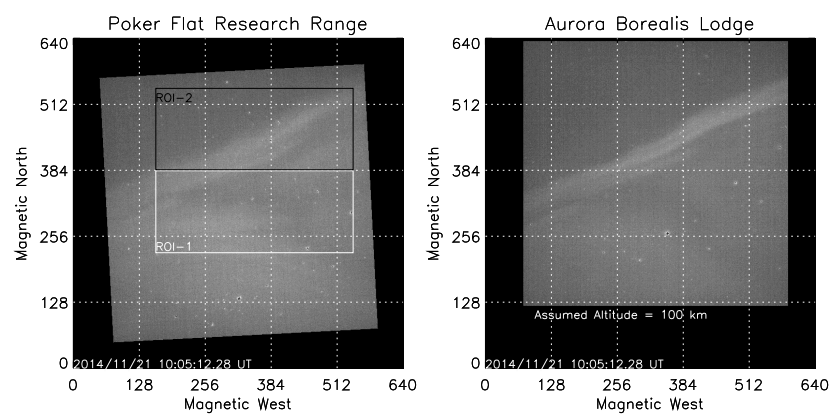

Figure 2. Example images of comparison. ROI-1 and ROI-2 are marked by white and black squares, respectively.

of view. Essentially the same method is therefore mostly applicable for many different types of aurora in general.

\section{Results and discussions}

Stereoscopic images of rapidly varying aurora were obtained at magnetic zenith just after the substorm onset at around 10:00 UT on 21 November 2014. In this paper we focus on the best $15 \mathrm{~s}$ time interval from 10:05 UT, in which a kind of rapidly varying "compound microstructures" (Kataoka et al., 2015) of pulsating patch and streaming arc coexist at the same time in both fields of view. Each camera had data gaps due to the unstable recording system at that time, but synchronized images were intermittently available for up to $5 \mathrm{~s}$ continuous time intervals as shown in Fig. 3. Examples of original images are shown in the Supplement in Movie 1 and Movie 2 for the first continuous time interval. It took tens of minutes for the $15 \mathrm{~s}$ data analysis using a standard PC, and a much faster method is needed for future real-time analysis.

Figure 3 shows the time variation of the auroral intensity and of emission altitude. Top panel shows that ROI-1 mainly contains rapidly pulsating patches while ROI-2 mainly contains streaming arcs. Many previous studies (e.g., Brown et al., 1976) suggest that pulsating patches and streaming arcs are caused by $10 \mathrm{keV}$ order and $1.0 \mathrm{keV}$ order electrons, respectively. For example, expected values of the main ionization altitudes of 40 and $4.0 \mathrm{keV}$ electrons are 85-95 and $110-130 \mathrm{~km}$, respectively (Tsurunen et al., 2009). In fact, the emission altitudes of the rapidly pulsating diffuse patch and streaming discrete arc were differentiated and estimated as 85-95 and 100-150 km, respectively as shown in bottom and middle panels in Fig. 3. Similar colors with those of peak cross-correlation coefficient are useful to see the error distribution, and typical error is estimated as $5-10 \mathrm{~km}$ as shown in Fig. 3, although the error of altitude estimation always depends on the spatial scale of aurora and selected ROI, etc.

These estimates of emission altitudes are consistent with those obtained in previous studies (e.g., Brown et al., 1976). It is therefore suggested that high-speed stereoscopy is useful to directly measure the emission altitudes of various types of rapidly varying aurora. The fact that two different altitudes 

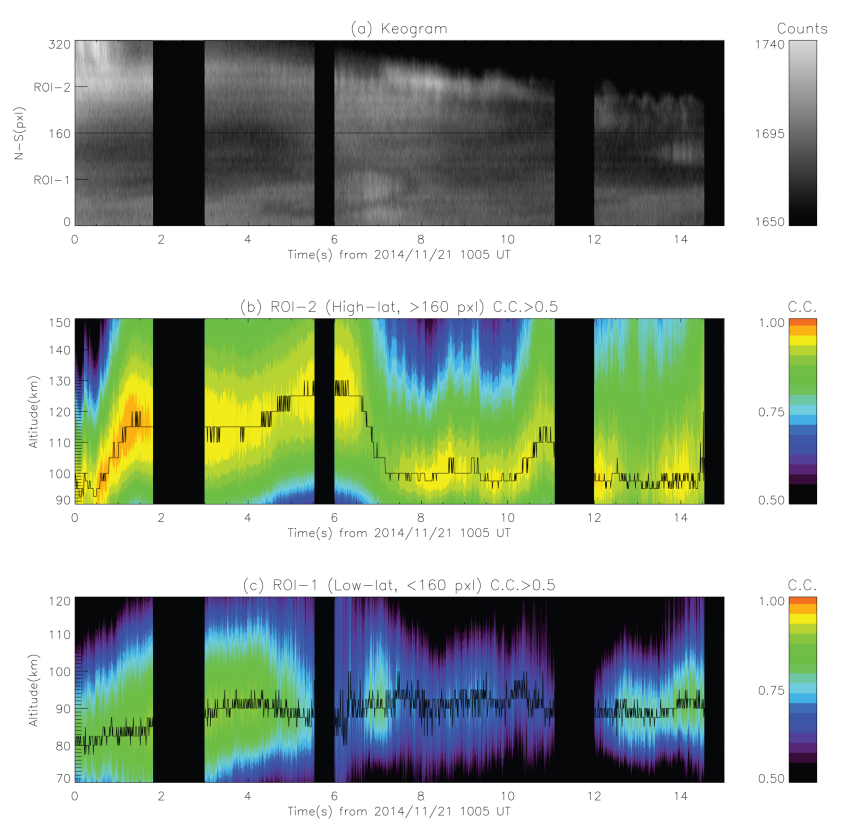

Figure 3. Time variation of the (a) auroral intensity and of (b, c) cross-correlation coefficient at each emission altitude. Top panel shows the north-south keogram across the center of starremoved images at PFRR, where grayscale shows the linearly increasing auroral intensity in arbitrary units. Middle and bottom panels show the altitude variations of aurora at high-latitude part ROI1 and low-latitude part ROI-2, respectively where blue to red colors show the linearly increasing cross-correlation coefficient (C.C.) from 0.5 to 1.0 . The altitudes of peak C.C. values are marked by black lines.

of rapidly varying auroras exist at the same time within a narrow field of view is consistent with the interpretation that "compound microstructures" (Kataoka et al., 2015) contain very different auroras at the same time within narrow field of view.

It is also found that the time variation of emission altitudes is relatively gradual (e.g., $10 \mathrm{~km}$ increase over $T=0.0-$ $5.0 \mathrm{~s})$ for pulsating patches and relatively fast $(20 \mathrm{~km}$ increase within $1 \mathrm{~s}$ after $T=0.5 \mathrm{~s}$ ) for streaming arcs, implying that the energization or de-energization timescale of pulsating patches and streaming arcs are very different, as originated from the magnetosphere and from the magnetosphereionosphere coupled region, respectively.

\section{Information about the Supplement}

Movie 1: example movie of half-speed playback obtained at Poker Flat Research Range.

Movie 2: example movie of half-speed playback obtained at Aurora Borealis Lodge.

\section{The Supplement related to this article is available online at doi:10.5194/angeo-34-41-2016-supplement.}

Acknowledgements. We thank Makoto Kumagai for his kind and patient support at Aurora Borealis Lodge. We thank Kevin Abnett for his technical help at Poker Flat Research Range. This study is supported by Grants-in-Aid for Scientific Research (25302006, 15H03732, 15H05815, and 15H05747) of Japan Society for the Promotion of Science (JSPS). This work was carried out by the joint research program of the Solar-Terrestrial Environment Laboratory, Nagoya University. The production of this paper was supported by an NIPR publication subsidy.

The topical editor, S. Milan, thanks one anonymous referee for help in evaluating this paper.

\section{References}

Brown, N. B., Davis, T. N., Hallinan, T. J., and Stanbaek-Nielsen, H. C.: Altitude of pulsating aurora determined by a new instrumental technique, Geophys. Res. Lett., 3, 403-404, 1976.

Dahlgren, H., Ivchenko, N., Sullivan, J., Lanchester, B. S., Marklund, G., and Whiter, D.: Morphology and dynamics of aurora at fine scale: first results from the ASK instrument, Ann. Geophys., 26, 1041-1048, doi:10.5194/angeo-26-1041-2008, 2008.

Frey, S., Frey, H. U., Carr, D. J., Bauer, O. H., and Haerendel, G.: Auroral emission profiles extracted from three-dimensionally reconstructed arcs, J. Geophys. Res., 101, 21731-21741, doi:10.1029/96JA01899, 1996.

Ivchenko, N., Blixt, E. M., and Lanchester, B. S.: Multispectral observations of auroral rays and curls, Geophys. Res. Lett., 32, L18106, doi:10.1029/2005GL022650, 2005.

Kataoka, R., Miyoshi, Y., Sakanoi, T., Yaegashi, A., Ebihara, Y., and Shiokawa, K.: Ground-based multispectral high-speed imaging of flickering aurora, Geophys. Res. Lett., 38, L14106, doi:10.1029/2011GL048317, 2011a.

Kataoka, R., Miyoshi, Y., Sakanoi, T., Yaegashi, A., Shiokawa, K., and Ebihara, Y.: Turbulent microstructures and formation of folds in auroral breakup arc, J. Geophys. Res., 116, A00K02, doi:10.1029/2010JA016334, 2011b.

Kataoka, R., Miyoshi, Y., Hampton, D., Ishii, T., and Kozako, H.: Pulsating aurora beyond the ultra-low-frequency range, J. Geophys. Res., 117, A08336, doi:10.1029/2012JA017987, 2012.

Kataoka, R., Miyoshi, Y., Shigematsu, K., Hampton, D., Mori, Y., Kubo, T., Yamashita, A., Tanaka, M., Takahei, T., Nakai, T., Miyahara, H., and Shiokawa, K.: Stereoscopic determination of all-sky altitude map of aurora using two groundbased Nikon DSLR cameras, Ann. Geophys., 31, 1543-1548, doi:10.5194/angeo-31-1543-2013, 2013.

Kataoka, R., Fukuda, Y., Miyoshi, Y., Miyahara, H., Itoya, S., Ebihara, Y., Hampton, D., Dahlgren, H., Whiter, D., and Ivchenko, N.: Compound auroral micromorphology: Groundbased high-speed imaging, Earth Planets Space, 67, 1-9, doi:10.1186/s40623-015-0190-6, 2015.

Miyoshi, Y., Katoh, Y., Nishiyama, T., Sakanoi, T., Asamura, K., and Hirahara, M.: Time of flight analysis of pulsating au- 
rora electrons, considering wave-particle interactions with propagating whistler mode waves, J. Geophys. Res., 115, A10312, doi:10.1029/2009JA015127, 2010.

Miyoshi, Y., Saito, S., Seki, K., Nishiyama, T., Kataoka, R., Asamura, K., Katoh, Y., Ebihara, Y., Sakanoi, T., Hirahara, M., Oyama, S., Kurita, S., and Santolik, O.: Relation between fine structure of energy spectra for pulsating aurora electrons and frequency spectra of whistler-mode chorus waves, J. Geophys. Res.Space, 120, 7728-7736, doi:10.1002/2015JA021562, 2015a.

Miyoshi, Y., Oyama, S., Saito, S., Kurita, S., Fujiwara, H., Kataoka, R., Ebihara, Y., Kletzing, C., Reeves, G., Santolik, O., Clilverd, M., Rodger, C. J., Turunen, E., and Tsuchiya F.: Energetic electron precipitation associated with pulsating aurora: EISCAT and Van Allen Probe observations. J. Geophys. Res.-Space, 120, 2754-2766, doi:10.1002/2014JA020690, 2015b.

Nishiyama, T., Sakanoi, T., Miyoshi, Y., Hampton, D. L., Katoh, Y., Kataoka, R., and Okano, S.: Multiscale temporal variations of pulsating auroras: On-off pulsation and a few $\mathrm{Hz}$ modulation, J. Geophys. Res.-Space, 119, 3514-3527, doi:10.1002/2014JA019818, 2014.

Stenbaek-Nielsen, H. and Hallinan, T.: Pulsating auroras: Evidence for noncollisional thermalization of precipitating electrons, J. Geophys. Res., 84, 3257-3271, doi:10.1029/JA084iA07p03257, 1979.
Stormer, C.: The Polar Aurora, Oxford University Press, Oxford, UK, 1955.

Turunen, E., Verronen, P. T., Seppala, A., Rodger, C. J., Clilverd, M. A., Tamminen, J., Enella, C.-F., and Ulicha, T.: Impact of different energies of precipitating particles on NOx generation in the middle and upper atmosphere during geomagnetic storms, J. Atmos. Sol.-Terr. Phy., 71, 1176-1189, 2009.

Whiter, D. K., Lanchester, B. S., Gustavsson, B., Ivchenko, N., and Dahlgren, H.: Using multispectral optical observations to identify the acceleration mechanism responsible for flickering aurora, J. Geophys. Res., 115, A12315, doi:10.1029/2010JA015805, 2010.

Whiter, D. K., Gustavsson, B., Partamies, N., and Sangalli, L.: A new automatic method for estimating the peak auroral emission height from all-sky camera images, Geosci. Instrum. Method. Data Syst., 2, 131-144, doi:10.5194/gi-2-131-2013, 2013.

Yaegashi, A., Sakanoi, T., Kataoka, R., Asamura, K., Miyoshi, Y., Sato, M., and Okano, S.: Spatial-temporal characteristics of flickering aurora as seen by high-speed EMCCD imaging observations, J. Geophys. Res., 116, A00K04, doi:10.1029/2010JA016333, 2011. 